\title{
Solubility of Carbon in Nanocrystalline $\alpha$-Iron
}

\author{
Alexander Kirchner and Bernd Kieback \\ Institute of Materials Science, Technische Universität Dresden, 01062 Dresden, Germany \\ Correspondence should be addressed to Alexander Kirchner, alexander.kirchner@tu-dresden.de
}

Received 24 February 2012; Accepted 3 May 2012

Academic Editor: Grégory Guisbiers

Copyright ( 12012 A. Kirchner and B. Kieback. This is an open access article distributed under the Creative Commons Attribution License, which permits unrestricted use, distribution, and reproduction in any medium, provided the original work is properly cited.

\begin{abstract}
A thermodynamic model for nanocrystalline interstitial alloys is presented. The equilibrium solid solubility of carbon in $\alpha$-iron is calculated for given grain size. Inside the strained nanograins local variation of the carbon content is predicted. Due to the nonlinear relation between strain and solubility, the averaged solubility in the grain interior increases with decreasing grain size. The majority of the global solubility enhancement is due to grain boundary enrichment however. Therefore the size effect on carbon solubility in nanocrystalline $\alpha$-iron scales with the inverse grain size.
\end{abstract}

\section{Introduction}

An enhancement of solid solubility has been found in many bulk nanocrystalline materials, for instance $[1,2]$. Often the bigger part of the effect can be attributed to nonequilibrium processing such as mechanical alloying [3]. However quantifying the impact of grain refinement on equilibrium solid solubility is important for understanding the behavior of nanocrystalline alloys upon thermal activation. In this context equilibrium shall be constrained by the assumption of a stable grain size.

The scope of this work is to develop a thermodynamic model of nanocrystalline alloys. It has to comprise both the nanoscale grains and the grain boundary regions, since the fraction of atoms located in those cannot be neglected. The description is based upon the previously published thermodynamic treatment of alloy grain boundaries [4] There the grain boundaries are approximated by a uniformly dilated lattice retaining the symmetry of the bulk material $[5,6]$ and are characterized by their volumetric strain $\Delta V / V_{0}$.

The interstitial solution of carbon in $\alpha$-iron is chosen as an alloy of practical relevance and experimental accessibility. In pure iron the nanocrystalline state is retained even after prolonged annealing at $650 \mathrm{~K}$ [7]. At this temperature and a time of $3600 \mathrm{~s}$, the diffusion length of carbon in bulk iron exceeds $50 \mu \mathrm{m}$ [8]. Since the carbon diffusivity will be larger in nanocrystalline iron, this enables chemical equilibration without strong grain growth.

\section{Thermodynamic Model of Nanocrystalline Fe-C}

The thermodynamic description of a binary Fe-C alloy under volumetric strain is based upon Kaufman and Schlosser's expression [9] for the Helmholtz free energy $F$. The free energy of the strained solid consists of $F^{\circ}$ at zero pressure and a second term that accounts for the elastic energy depending on the parameter $\chi=\left(V / V_{0}\right)^{1 / 3}$ using the Vinet et al. universal equation of state [10]. The isothermal bulk modulus $B_{0}$, the molar volume $V_{0}$, and the anharmonicity parameter $\eta_{0}$ are given at zero pressure:

$$
F=F^{\circ}+\frac{9 B_{0} V_{0}}{\eta_{0}^{2}}\left[\left\{\eta_{0}(1-\chi)-1\right\} e^{\eta_{0}(1-\chi)}+1\right] .
$$

A two-sublattice model $\mathrm{Fe}(\mathrm{Va}, \mathrm{C})_{3}$ is used to describe the interstitial solution of carbon in $\alpha$-iron. In the second sublattice carbon substitutes normally vacant octahedral sites. Here $y_{\mathrm{C}}$ marks the molar fraction of carbon in the sublattice. Its relation to the carbon content given as the molar fraction $x_{\mathrm{C}}$ is $y_{\mathrm{C}}=x_{\mathrm{C}} /\left(3\left(1-x_{\mathrm{C}}\right)\right)$. Conveniently choosing pure $\alpha$-iron and graphite as the reference state, 
the molar free energy $F^{\circ}$ of the unstrained alloy is stated by [11] in units of Joule where $T$ is the temperature in Kelvin:

$$
\begin{aligned}
F^{\circ}= & (322050+75.667 T) y_{\mathrm{C}} \\
& +3 R T\left(y_{\mathrm{C}} \ln y_{\mathrm{C}}+\left(1-y_{\mathrm{C}}\right) \ln \left(1-y_{\mathrm{C}}\right)\right) \\
& -190 T\left(1-y_{\mathrm{C}}\right) y_{\mathrm{C}} .
\end{aligned}
$$

Assuming a dilute alloy, $B_{0}$ and $\eta_{0}$ of pure $\alpha$-iron are used in (1). Values of $B_{0}=178.6 \mathrm{GPa}, V_{0}=7.09 \cdot 10^{-6} \mathrm{~m}^{3} / \mathrm{mol}$ [12], and $\eta_{0}=5.16$ [13] at $T=298 \mathrm{~K}$ are employed. Their temperature dependence is calculated according to Vinet and coworkers [10] using the coefficient of volumetric thermal expansion $\alpha_{0}=36.9 \cdot 10^{-6} \mathrm{~K}^{-1}$ [12]. At low solute concentration the molar volume $V_{0}$ of the alloy varies linearly with $x_{\mathrm{C}}$, and

$$
\chi=\sqrt[3]{\frac{1+\Delta V / V_{0}}{1+x_{\mathrm{C}} \Omega_{\mathrm{C}}^{\mathrm{Fe}, \mathrm{bcc}}}}
$$

is obtained. The volume size factor of carbon in $\alpha$-iron $\Omega_{\mathrm{C}}^{\mathrm{Fe}, \mathrm{bcc}}=0.825$ is averaged from two publications $[14,15]$. The equilibrium between carbon in strained $\alpha$-iron and the reference state graphite is given by $F+\left(1-x_{\mathrm{C}}\right) \cdot\left(\partial F / \partial x_{\mathrm{C}}\right)=0$.

The strain of the crystallite interior is calculated using Weissmüller's model [16]. Accordingly local strain in bulk nanocrystalline materials is caused by the fact that the cavities defined by the adjacent grains need to accommodate a finite number of lattice planes. The maximum linear strain $\varepsilon_{\max }$ is given by the interatomic distance $r_{\mathrm{NN}}$ (for iron $r_{\mathrm{NN}}=$ $0.252 \mathrm{~nm}[12]$ ) and the grain size $D$ :

$$
\left|\varepsilon_{\max }\right|=\frac{1}{4} \sqrt{\frac{3}{2}} \frac{r_{\mathrm{NN}}}{D} .
$$

The predicted root mean square strain is in good agreement with strain measured in nc-Fe by X-ray diffraction $[17,18]$. Here the mean strain $\langle\varepsilon\rangle_{V}=-2\langle f\rangle_{A} /\left(3 B_{0} D\right)$ is neglected, because with a grain-boundary stress $\langle f\rangle_{A}$ of $1.1 \mathrm{~N} / \mathrm{m}$ [19] it is more than a magnitude of order smaller than $\left|\varepsilon_{\max }\right|$. The volumetric strain is given by the superposition of the linear strain in three dimensions $\Delta V / V_{0} \cong \varepsilon_{x}+\varepsilon_{y}+\varepsilon_{z}$.

Furthermore a thickness $w$ of $0.7 \mathrm{~nm}$ representing 3-4 monolayers and a value of $\Delta V / V_{0}=0.12$ is used to describe average grain boundaries. This choice of parameters yields a good agreement with an experimental value of the interface free energy of $468 \mathrm{~mJ} / \mathrm{m}^{2}$ at $1723 \mathrm{~K}$ [20].

Finally the resulting global solubility $x_{C}^{\text {global }}$ is calculated from the grain boundary and interior compositions with their respective molar fractions as weighting factors:

$$
\begin{gathered}
x_{C}^{\text {global }}=f x_{C}^{\mathrm{GB}}+(1-f) \bar{x}_{C}^{\text {interior }}, \\
f=\frac{(D+w)^{3}-D^{3}}{(D+w)^{3}+D^{3}\left(\Delta V / V_{0}\right)} .
\end{gathered}
$$

The molar fraction of the atoms located in grain boundaries $f$ is corrected for the different atomic densities and converges for $\Delta V / V_{0}=0$ and large grain sizes toward $3 w / D$.

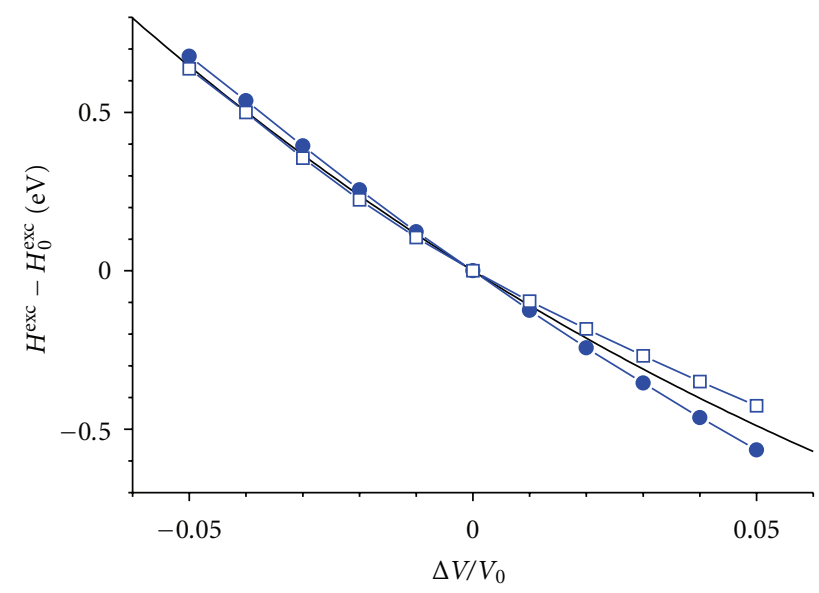

FIgURE 1: Difference of the excess enthalpies of a carbon atom in strained and strain-free iron. Results from [21] are marked as filled circles (DFT) and open squares (MEAM).

\section{Results and Discussion}

Recently $a b$ initio methods based on density-functional theory (DFT) allow for highly accurate simulation of carbon solution in $\alpha$-iron. The excess enthalpy of carbon in iron as defined by [21] depending on volumetric strain was calculated at $T=0 \mathrm{~K}$ and infinite carbon dilution. The result is presented in Figure 1. Good agreement with the strain dependence of excess enthalpies computed by DFT and a modified embedded-atom method (MEAM) is observed. The calculated derivative of the excess enthalpy with respect to the atomic volume at $\Delta V / V_{0}=0$ is $-0.95 \mathrm{eV} / \AA^{3}$ as compared to $-1.08 \mathrm{eV} / \AA^{3}$ (DFT) and $-0.84 \mathrm{eV} / \AA^{3}$ (MEAM).

The maximum linear strain of the grain interior given by (4) can be used to calculate the local volumetric strain assuming three independent axes. For a grain size of $20 \mathrm{~nm}$ the linear strain varies locally between $-3.8 \cdot 10^{-3}$ and $+3.8 \cdot 10^{-3}$ and the volumetric strain between -0.011 and +0.011 with the frequency distribution shown in Figure 2 . Close to $\Delta V / V_{0}=0$ the calculated carbon concentration in equilibrium with graphite at $673 \mathrm{~K}$ coincides with the linear theory of thermochemical equilibrium of solids under stress [22]. For large strains nonlinear behavior is observed. At lower values of $\Delta V / V_{0}$ indicating strong compression, the carbon concentration converges towards zero, which is the physically reasonable behavior.

The expected variation in composition between individual nanograins can be deduced from the range of values calculated for $x_{\mathrm{C}}^{\text {interior }}$. For a grain size of $20 \mathrm{~nm}$ the ratio between the maximum $\left(9.8 \cdot 10^{-4}\right.$ at.\%) and the minimum carbon concentration $\left(1.7 \cdot 10^{-5}\right.$ at.\%) is 59. This ratio will be even larger for smaller grain sizes. Another notable consequence of the nonlinear trend of $x_{C}^{\text {interior }}$ is that weighted averaging yields a deviation from bulk solubility despite the strain distribution symmetry. Figure 3 shows the average carbon solubility in the grain interior as a function of inverse grain size. It increases with decreasing grain size slightly. 


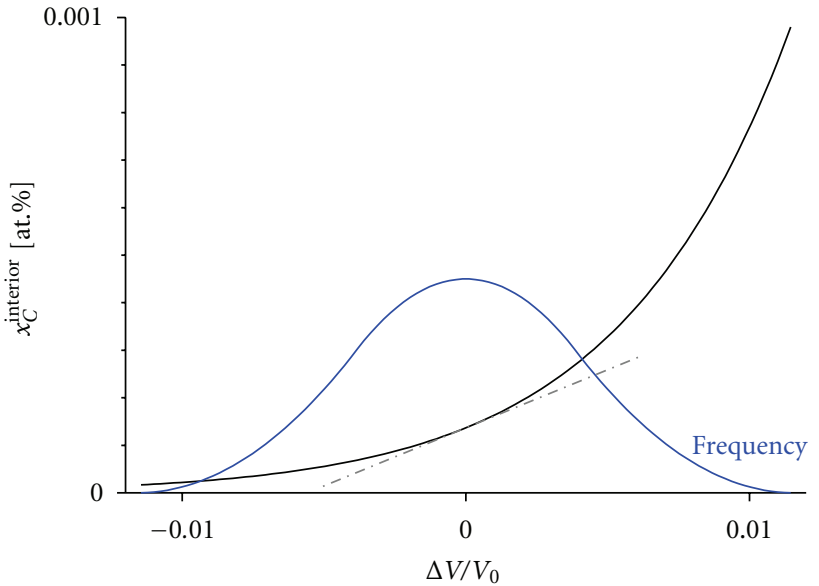

FIGURE 2: Solubility of carbon in the strained grain interior at $673 \mathrm{~K}$. The range and frequency distribution of the local strain is calculated for a grain size of $20 \mathrm{~nm}$. The broken line represents the result of the theory by Larché and Cahn [22].

$\bar{x}_{C}^{\text {interior }}$ is doubled with respect to the bulk solubility for $11 \mathrm{~nm}$ grains.

The carbon concentration in grain boundaries was calculated in equilibrium with graphite assuming it to be independent of curvature and grain size. At a temperature of $673 \mathrm{~K}$ the computed value of $x_{\mathrm{C}}^{\mathrm{GB}}$ is 3.9 at.\%. This means that carbon is enriched by a factor of $3 \cdot 10^{4}$ in the grain boundaries with respect to the bulk. Atom probe microscopy measurements confirm the presence of a minimum of 2 at.\% carbon in $\alpha$-iron grain boundaries [23]. Other experiments at $873 \mathrm{~K}[24,25]$ yielded higher values, expressed as excess densities of carbon at the interface of $\Gamma_{\mathrm{C}}^{\mathrm{GB}} \approx 20 \mu \mathrm{mol} / \mathrm{m}^{2}$. The corresponding theoretical prediction is lower at $\Gamma_{\mathrm{C}}^{\mathrm{GB}}=$ $5 \mu \mathrm{mol} / \mathrm{m}^{2}$. The difference may be explained by the strong variation between individual grain boundaries and the information depth of the analytical methods employed. In the case of autoradiography it exceeds the grain boundary thickness of approximately $1 \mathrm{~nm}$ by far. The detected amount is likely to include carbon enriched at stress fields around grain boundaries as well. Then the concentration in the grain boundary core is lower than $\Gamma_{\mathrm{C}}^{\mathrm{GB}}$ suggests.

Calculating the global solubility according to (5) yields an almost linear relation with the inverse grain size as Figure 3 illustrates. The reason is that the contribution of enriched grain boundaries dominates the size effect. Figure 4 shows the calculated solubility of carbon in nanocrystalline $\alpha$-iron of various grain sizes. Thereafter a pronounced increase in equilibrium carbon solubility is to be expected in nanocrystalline iron in comparison to bulk iron.

\section{Conclusions}

A thermodynamic model for nanocrystalline $\alpha$-phase Fe-C alloys has been presented. Considering the strained grain interior in equilibrium with graphite local variation of the carbon concentration but only a weak size effect on the average carbon solubility is found. While the extent of

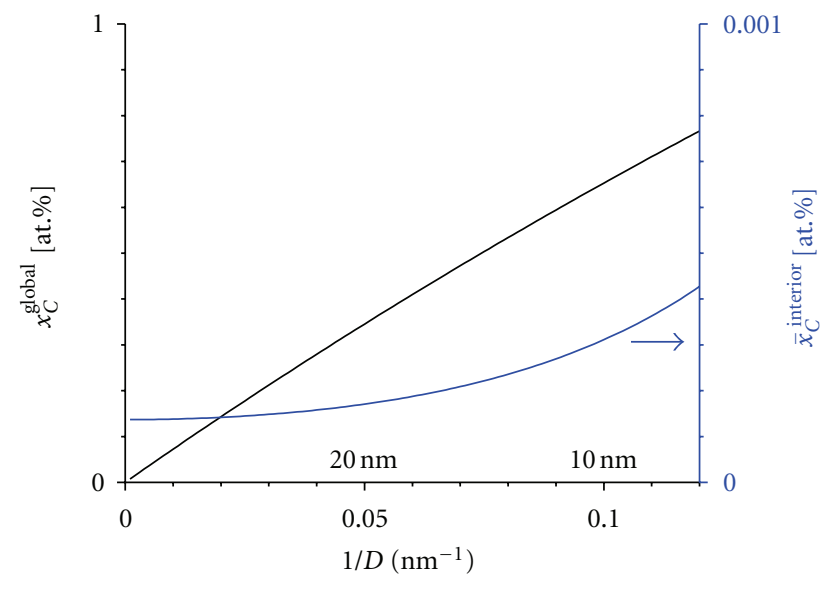

FIgURE 3: Average solubility of carbon in the grain interior $\bar{x}_{C}^{\text {interior }}$ and resulting solubility $x_{\mathrm{C}}^{\text {global }}$ in nanocrystalline $\alpha$-iron at $673 \mathrm{~K}$ as a function of grain size. Note that the axis of $\bar{x}_{C}^{\text {interior }}$ is scaled by a factor 1000 .

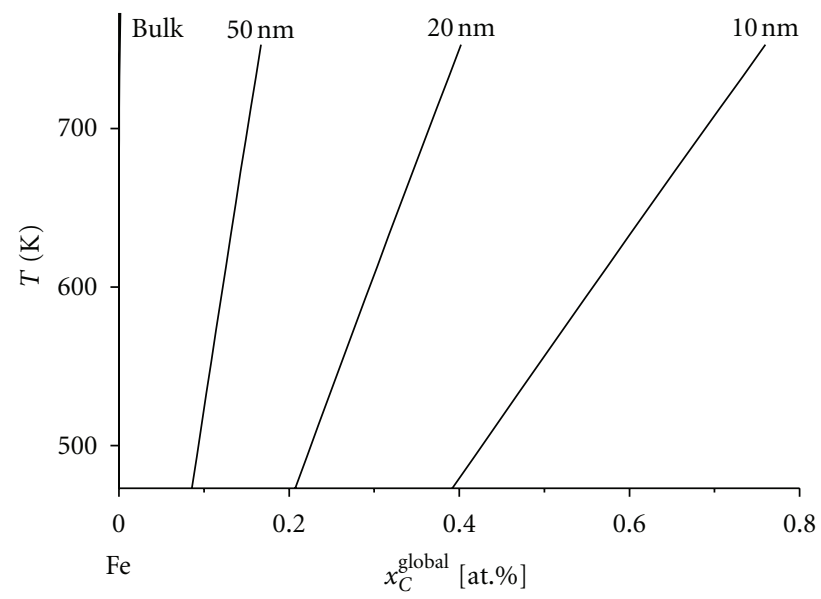

FIGURE 4: Calculated solvus lines of carbon in nanocrystalline $\alpha$ iron of 10, 20, and $50 \mathrm{~nm}$ grain size compared to bulk iron.

enrichment at grain boundaries is not precisely established, grain boundary segregation dominates the solubility increase in the nanocrystalline state. It is concluded that the excess carbon solubility in nanocrystalline iron over bulk iron is proportional to the inverse grain size. At a given temperature the overall solubility follows

$$
x_{\mathrm{C}}^{\text {global }} \approx x_{\mathrm{C}}^{\text {bulk }}+\frac{\text { const }}{D}
$$

\section{Acknowledgments}

The research of A. Kirchner was supported by Deutsche Forschungsgemeinschaft via the Emmy Noether Programme. 


\section{References}

[1] H. Gleiter, "Nanocrystalline solids," Journal of Applied Crystallography, vol. 24, no. 2, pp. 79-90, 1991.

[2] C. D. Terwilliger and Y. M. Chiang, "Size-dependent solute segregation and total solubility in ultrafine polycrystals: Ca in $\mathrm{TiO}_{2}$," Acta Metallurgica Et Materialia, vol. 43, no. 1, pp. 319 328, 1995.

[3] C. Suryanarayana, "Mechanical alloying and milling," Progress in Materials Science, vol. 46, no. 1-2, pp. 1-184, 2001.

[4] A. Kirchner and B. Kieback, "Thermodynamic model of alloy grain boundaries," Scripta Materialia, vol. 64, no. 5, pp. 406409, 2011.

[5] H. J. Fecht, "Intrinsic instability and entropy stabilization of grain boundaries," Physical Review Letters, vol. 65, no. 5, pp. 610-613, 1990.

[6] M. Wagner, "Structure and thermodynamic properties of nanocrystalline metals," Physical Review B, vol. 45, no. 2, pp. 635-639, 1992.

[7] T. R. Malow and C. C. Koch, "Grain growth in nanocrystalline iron prepared by mechanical attrition," Acta Materialia, vol. 45, no. 5, pp. 2177-2186, 1997.

[8] E. A. Brandes and G. B. Brook, Eds., Smithells Metals Reference Book, Butterworth Heinemann, Oxford, UK, 1999.

[9] M. Kaufman and H. Schlosser, "A thermodynamic model for pressurized solids," Journal of Physics, vol. 7, no. 11, article 03, pp. 2259-2264, 1995.

[10] P. Vinet, J. R. Smith, J. Ferrante, and J. H. Rose, "Temperature effects on the universal equation of state of solids," Physical Review B, vol. 35, no. 4, pp. 1945-1953, 1987.

[11] P. Gustafson, "A thermodynamic evaluation of the Fe-C system," Scandinavian Journal of Metallurgy, vol. 14, no. 5, pp. 259-267, 1985.

[12] W. Martienssen and H. Warlimont, Eds., Handbook of Condensed Matter and Materials Data, Springer, Berlin, Germany, 2005.

[13] J. H. Rose, J. R. Smith, F. Guinea, and J. Ferrante, "Universal features of the equation of state of metals," Physical Review B, vol. 29, no. 6, pp. 2963-2969, 1984.

[14] E. J. Fasiska and H. Wagenblast, "Dilation of alpha iron by carbon," Transactions of the Metallurgical Society of AIME, vol. 239, no. 11, pp. 1818-1820, 1967.

[15] H. W. King, "Quantitative size-factors for interstitial solid solutions," Journal of Materials Science, vol. 6, no. 9, pp. 11571167, 1971.

[16] J. Weissmüller, "Thermodynamics of nanocrystalline solids," in Nanocrystalline Metals and Oxides, P. Knauth and J. Schoonman, Eds., Kluwer Academic, New York, NY, USA, 2002.

[17] E. Bonetti, L. Del Bianco, L. Pasquini, and E. Sampaolesi, "Thermal evolution of ball milled nanocrystalline iron," Nanostructured Materials, vol. 12, no. 5, pp. 685-688, 1999.

[18] Y. H. Zhao, H. W. Sheng, and K. Lu, "Microstructure evolution and thermal properties in nanocrystalline Fe during mechanical attrition," Acta Materialia, vol. 49, no. 2, pp. 365375, 2001.

[19] P. Zimmer and R. Birringer, "Measuring the interface stress of nanocrystalline iron," Applied Physics Letters, vol. 92, no. 8, Article ID 081912, 3 pages, 2008.

[20] L. E. Murr, Interfacial Phenomena in Metals and Alloys, Addison-Wesley, New York, NY, USA, 1975.

[21] E. Hristova, R. Janisch, R. Drautz, and A. Hartmaier, "Solubility of carbon in $\alpha$-iron under volumetric strain and close to the $\Sigma 5(310)[001]$ grain boundary: comparison of DFT and empirical potential methods," Computational Materials Science, vol. 50, no. 3, pp. 1088-1096, 2011.

[22] F. Larché and J. W. Cahn, "A linear theory of thermochemical equilibrium of solids under stress," Acta Metallurgica, vol. 21, no. 8, pp. 1051-1063, 1973.

[23] A. Atrens, J. Q. Wang, K. Stiller, and H. O. Andren, "Atom probe field ion microscope measurements of carbon segregation at an $\alpha: \alpha$ grain boundary and service failures by intergranular stress corrosion cracking," Corrosion Science, vol. 48, no. 1, pp. 79-92, 2006.

[24] J. M. Papazian and D. N. Besherb, "Grain boundary segregation of carbon in iron," Metallurgical Transactions, vol. 2, no. 2, pp. 497-503, 1971.

[25] H. Hänsel and H. J. Grabke, "Grain boundary segregation of phosphorus and carbon in ferritic iron," Scripta Metallurgica, vol. 20, no. 11, pp. 1641-1644, 1986. 

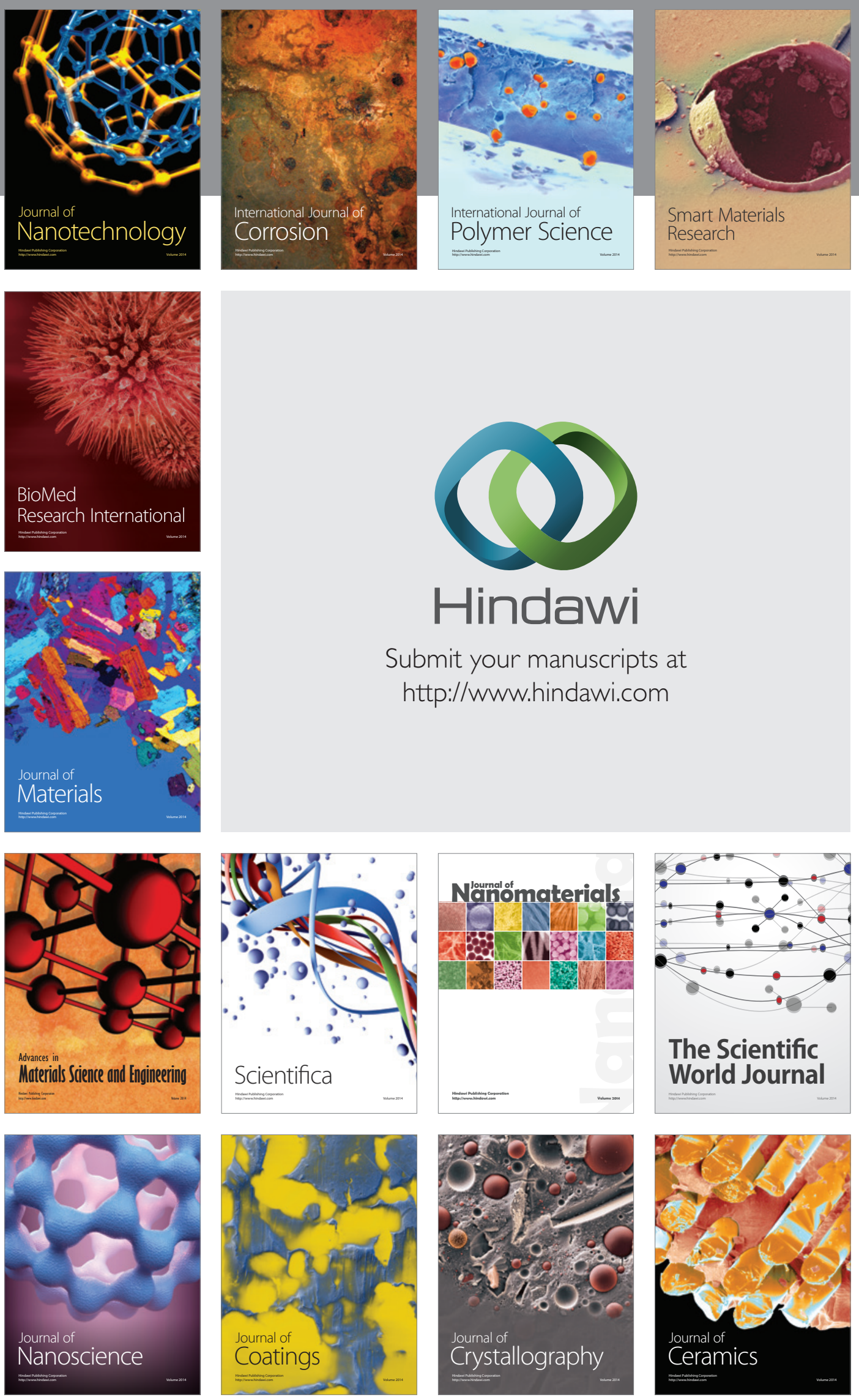

The Scientific World Journal

Submit your manuscripts at

http://www.hindawi.com

\section{World Journal}

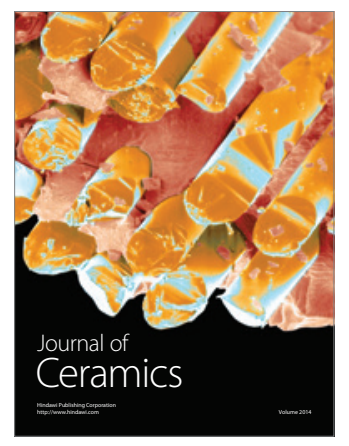

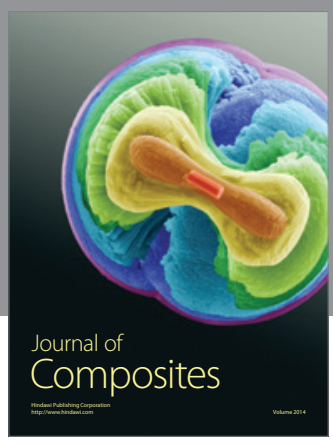
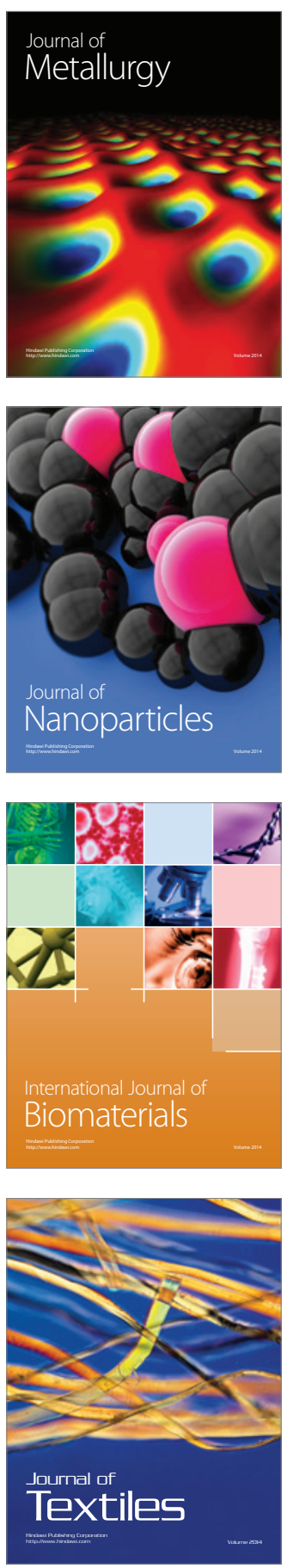